\title{
Validity and reliability of an online self-report 24-h dietary recall method (Intake24): a doubly labelled water study and repeated-measures analysist
}

\author{
Emma Foster ${ }^{1}$, Clement Lee $^{2,3} \mathbb{C}$, Fumiaki Imamura ${ }^{4}$, Stefanie E. Hollidge ${ }^{4}$, Kate L. Westgate ${ }^{4}$, \\ Michelle C. Venables ${ }^{5}$, Ivan Poliakov ${ }^{6}$, Maisie K. Rowland ${ }^{1}$, Timur Osadchiy ${ }^{6}$, Jennifer C. Bradley ${ }^{1}$ (D), \\ Emma L. Simpson ${ }^{6}$, Ashley J. Adamson ${ }^{1}$, Patrick Olivier ${ }^{7}$, Nick Wareham ${ }^{4}$, Nita G. Forouhi ${ }^{4}$ and \\ Soren Brage ${ }^{4}$ \\ ${ }^{1}$ Human Nutrition Research Centre, Institute of Health and Society, Newcastle University, Newcastle upon Tyne, UK \\ ${ }^{2}$ School of Mathematics, Statistics and Physics, Newcastle University, Newcastle upon Tyne, UK \\ ${ }^{3}$ Department of Mathematics and Statistics, Lancaster University, Lancaster, UK \\ ${ }^{4} \mathrm{MRC}$ Epidemiology Unit, University of Cambridge, Cambridge, UK \\ ${ }^{5} \mathrm{MRC}$ Elsie Widdowson Laboratory, Cambridge, UK \\ ${ }^{6}$ Open Lab, School of Computing Science, Newcastle University, Newcastle upon Tyne, UK \\ ${ }^{7}$ Faculty of Information Technology, Monash University, Clayton, VIC, Australia
}

(Received 2 April 2019 - Final revision received 8 June 2019 - Accepted 13 June 2019)

Journal of Nutritional Science (2019), vol. 8, e29, page 1 of 9

doi:10.1017/jns.2019.20

\section{Abstract}

Online self-reported 24-h dietary recall systems promise increased feasibility of dietary assessment. Comparison against interviewer-led recalls established their convergent validity; however, reliability and criterion-validity information is lacking. The validity of energy intakes (EI) reported using Intake24, an online 24-h recall system, was assessed against concurrent measurement of total energy expenditure (TEE) using doubly labelled water in ninety-eight UK adults (40-65 years). Accuracy and precision of EI were assessed using correlation and Bland-Altman analysis. Test-retest reliability of energy and nutrient intakes was assessed using data from three further UK studies where participants (11-88 years) completed Intake24 at least four times; reliability was assessed using intra-class correlations (ICC). Compared with TEE, participants under-reported EI by $25 \%$ ( $95 \%$ limits of agreement $-73 \%$ to $+68 \%$ ) in the first recall, $22 \%(-61 \%$ to $+41 \%)$ for average of first two, and $25 \%(-60 \%$ to $+28 \%)$ for first three recalls. Correlations between EI and TEE were $0 \cdot 31$ (first), $0 \cdot 47$ (first two) and 0.39 (first three recalls), respectively. ICC for a single recall was 0.35 for EI and ranged from 0.31 for Fe to 0.43 for non-milk extrinsic sugars (NMES). Considering pairs of recalls (first two $v$. third and fourth recalls), ICC was 0.52 for EI and ranged from 0.37 for fat to 0.63 for NMES. EI reported with Intake24 was moderately correlated with objectively measured TEE and underestimated on average to the same extent as seen with interviewer-led 24 -h recalls and estimated weight food diaries. Online 24-h recall systems may offer low-cost, low-burden alternatives for collecting dietary information.

Key words: Dietary assessment: Online 24-h dietary recall: Doubly labelled water: Validation: Repeatability: Reliability: UK adults

\footnotetext{
* Corresponding author: Emma Simpson, email Emma.Simpson@ncl.ac.uk
}

Abbreviations: ASA $24^{\circledR}$, automated self-administered 24-h dietary assessment tool; DLW, doubly labelled water; EI, energy intake; ICC, intra-class correlation coefficient; Intake24, self-completed computerised dietary recall system based on multiple-pass 24-hour recall; NMES, non-milk extrinsic sugars; RQ, respiratory quotient; TEE, total energy expenditure.

†The original version of this article was published with an incorrect author affiliation. A notice detailing this has been published and the error rectified in the online PDF and HTML copies. 
Information on dietary intakes of individuals and populations is important in determining diet-disease associations, identifying deficiencies and excesses of nutrients, and evaluating the impact of interventions. The majority of methods for assessing the diet of individuals involve an interview with trained personnel, manual coding of foods, calculation of portion sizes, and matching to food composition tables. Therefore, such methods tend to be costly and time-consuming. With traditional methods, such as the weighed food diary, issues of compliance and under-reporting of habitual energy intake $(\mathrm{EI})^{(1,2)}$, participant selection bias and recording bias ${ }^{(3)}$ are a significant concern.

Recent advances in technology and the ubiquity of Internet access in many countries have led to the development of web-based systems for collecting information on dietary intake remotely. These include online dietary $24-\mathrm{h}$ recall systems $^{(4,5)}$, online food diaries ${ }^{(6)}$ and online $\mathrm{FFQ}^{(7)}$. These systems can be completed at a time and place convenient to the participant, without the need for a trained interviewer, and this may reduce the respondent burden and reduce barriers to participation.

Intake24 is an online dietary recall system (https://intake24. co.uk/) which can be completed by participants remotely. Originally designed for use by people aged 11-24 years it was subsequently extended for the general adult population and tested with people aged $11-88$ years $^{(8)}$. The system is based on the multiple-pass $24-\mathrm{h}$ recall $^{(9)}$ and contains a database of over 2500 foods linked to food composition codes $^{(10)}$. Versions are available for the UK, Portugal, Denmark, New Zealand and the United Arab Emirates, with versions for India and Australia under development. A series of food photographs are used for portion size estimation. These have previously been criterion-validated in a feeding study and also evaluated for convergent validity against weighed food diaries with children aged 18 months to 16 years and their parents ${ }^{(11,12)}$. Intake24 was developed through four cycles of user-testing and feedback ${ }^{(13)}$. Convergent validity testing of Intake 24 against interviewer-led 24-h recalls found that the two methods yielded comparable estimates $^{(14)}$; however, the instrument has not yet been criterion-validated against objective measures of energy, nor has reliability been examined.

The doubly labelled water (DLW) method is considered the reference standard to estimate free-living total energy expenditure (TEE) ${ }^{(15,16)}$; one of its uses has been to validate dietary EI instruments. The underlying assumption is that if participants are in energy balance, then over a period of time, total EI should be equivalent to $\mathrm{TEE}^{(17)}$. These comparisons have led to the observation that underestimation of food intakes is a common problem in dietary surveys $^{(3,18)}$.

To establish the validity and reliability of the system for use in UK adults, we aimed to: (1) assess the validity of self-reported EI using Intake24 in a cohort of adults against concurrent objective measures of energy expenditure using DLW; and (2) test the reliability of estimates of energy and key nutrients using pooled data from studies for participants completing four or more recalls.

\section{Methods}

Validation of Intake24 reported energy intake against doubly labelled water measured energy expenditure

Study population and recruitment. We recruited fifty men and fifty women across three age categories (40-49 years; 50-59 years; 60-65 years) across a wide range of BMI from the Fenland Study, an ongoing population-based study in the Cambridgeshire area, $\mathrm{UK}^{(19)}$. A sample size of 100 participants was recruited on a first-come, first-served basis when fulfilling age/sex/BMI category eligibility and asked to attend two clinic visits. This size of sample allows estimation of the $95 \%$ CI about $\pm 0 \cdot 34 s$ (where $s$ is the standard deviation of the differences between measurements by the two methods) ${ }^{(20)}$. Travel expenses were paid but participants did not otherwise receive any monetary incentive for taking part. Data collection for this component of the study was carried out between November 2015 and September 2016. See Supplementary Fig. S1 for the participant flow chart for the validation study. The present study was conducted according to the guidelines laid down in the Declaration of Helsinki. Ethical approval for the study was obtained from Cambridge University Human Biology Research Ethics Committee (reference no. HBREC/2015.16) and all participants provided written informed consent.

Doubly labelled water administration. Participants attended their first clinic visit with a (baseline) urine sample collected at least $1 \mathrm{~d}$ prior to their visit (sample bottles provided in their appointment letter). During this visit, a second baseline (fasting) urine sample was collected. Participants were then asked to drink a body weight-specific dose of DLW (deuterium oxide-18; $\mathrm{D}_{2}^{18} \mathrm{O}$ ) and collect daily (post-dose) urine samples for the next 9-10 d. The dose used was $174 \mathrm{mg} / \mathrm{kg} \mathrm{H} \mathrm{H}_{2}^{18} \mathrm{O}$ and $70 \mathrm{mg} / \mathrm{kg}^{2} \mathrm{H}_{2} \mathrm{O}$. (Oxygen 18 was supplied by Sercon Ltd; deuterium was supplied by Goss Scientific Instruments Ltd, the UK distributor for Cambridge Isotope Laboratories.) The method of Schoeller was followed which fixes the space ratio to a value of $1.03^{16}$.

Participants were provided with labelled sampling bottles and a recording sheet and were instructed to collect one urine sample every day, at a similar time of day, at any time apart from the first void of the day. Participants were asked to record the date and time of each sample and keep the samples refrigerated until returning them at the second clinic visit following the free-living observation period. A final post-dose urine sample was obtained during the second clinic visit. All participants provided enough pre- and post-dose samples for calculation of TEE (see below). Height $(\mathrm{cm})$ and weight $(\mathrm{kg})$ were measured using standardised anthropometric procedures and BMI was calculated $\left(\mathrm{kg} / \mathrm{m}^{2}\right)$.

Intake24 administration. Participants were asked to complete Intake24 at least twice and ideally on three occasions during the DLW measurement period but the days on which to complete the recall were not specified. At the first clinic visit, each participant was issued with a 
unique username and password and provided with the URL (i. e. web address) with which they could access Intake24. If the participant had not completed at least two instances of Intake24 during the measurement period, or did not have Internet access, they were asked to complete Intake24 at the second clinic visit. Two participants did not complete Intake24 remotely. One of whom provided dietary data on paper at the second visit; these two individuals were excluded from this analysis.

Doubly labelled water sample analysis. Urine samples were analysed, in duplicate, for ${ }^{18} \mathrm{O}$ enrichment using the $\mathrm{CO}_{2}$ equilibration method of Roether ${ }^{(21)}$. Briefly, $0.5 \mathrm{ml}$ of sample was transferred into $12 \mathrm{ml}$ vials (Labco Ltd), flush-filled with $5 \% \mathrm{CO}_{2}$ in $\mathrm{N}_{2}$ gas and equilibrated overnight whilst agitated on rotators (Stuart, Bibby Scientific). Headspace of the samples was then analysed using a continuous flow isotope ratio mass spectrometer (AP2003; Analytical Precision Ltd). For ${ }^{2} \mathrm{H}$ enrichment, $0.4 \mathrm{ml}$ of sample was flush-filled with $\mathrm{H}_{2}$ gas and equilibrated over 6 $h$ in the presence of a platinum catalyst. Headspace of the samples was then analysed using a dual-inlet isotope ratio mass spectrometer (Isoprime; GV Instruments).

All samples were measured alongside secondary reference standards previously calibrated against the primary international standards Vienna-Standard Mean Ocean Water (vSMOW) and Vienna-Standard Light Antarctic Precipitate (vSLAP) (International Atomic Energy Agency). Sample enrichments were corrected for interference according to Craig $^{(22)}$ and expressed relative to vSMOW. Analytical precisions are better than $\pm 0.62 \%$ for $\delta^{18} \mathrm{O}$ and $\pm 0.5 \%$ for $\delta^{2} \mathrm{H}$. Please see Supplementary Calculation S1 for full details.

Data analysis. The method of Bland \& Altman ${ }^{(20)}$ was used to examine accuracy (mean bias) and precision (root mean square error and $95 \%$ limits of agreement) of reported EI by Intake24 against TEE measured using DLW. The ratio of reported daily EI (based on the first 24-h recall, the mean of the first two 24-h recalls and the mean of the first three 24-h recalls) to energy expenditure was calculated. As the data were not normally distributed, they were log-transformed. We define absolute validity by the $\log$-ratio $(\log (\mathrm{EI} / \mathrm{TEE}))$, where a negative log-ratio represents under-reporting and a positive $\log$-ratio indicates over-reporting of EI. The ratio of the arithmetic mean is also presented along with the geometric mean to allow comparison with previous studies.

We also examined the correlation between reported EI and energy expenditure to quantify ability of the instrument to rank individuals. In addition, we examined the role of intraindividual intake variation in these correlation coefficients using data for participants who had reported at least $3 \mathrm{~d}$, as described by Rimm et al. ${ }^{(23)}$. To assess whether the validity of Intake24 depended on demographic characteristics, we applied a mixed-effects model to account for multiple observations per individual, in which the dependent variable was the log-ratio, and the covariates included age, sex, height (in $\mathrm{cm})$ and BMI.

\section{Assessment of Intake24 reliability}

Study population and recruitment. The repeatability of measures of EI and key nutrients was examined using datasets from three previous surveys. These were a comparison of Intake24 against interviewer-led recalls in 11- to 24-year-olds (survey $1 ; n$ 129) ${ }^{(14)}$, comparison of Intake24 against interviewer-led recalls in adults aged 24-68 years (survey $2 ; n$ 46) and a field test of Intake24 in the Scottish Health Survey population with people aged 11-88 years old (survey $3 ; n$ 133) ${ }^{(8)}$. See Supplementary Fig. S2 for the participant flow chart for the repeatability study.

Only the initial mode of contact differed between the surveys. For survey 1, 11- to 16-year-olds were recruited from secondary schools in Dundee and Newcastle upon Tyne. The 17- to 24-year-olds were recruited by a recruitment agency who approached potential participants in the street. For survey 2 , posters and leaflets were displayed in locations around Newcastle including the University campus, local shops, fitness centres and childcare facilities. Recruitment for survey 3 was conducted in collaboration with ScotCen Social Research; 1000 participants who had previously taken part in the Scottish Health Survey were sent an introductory letter and followed up by telephone.

All participants were required to give written consent (written assent and parental consent were obtained for those under the age of 18 years) before participating in the research. Ethical approval for these surveys was granted by Newcastle University's Faculty of Medical Sciences Ethics Committee (reference no. 00706/2013, survey 1; no. 01018/2016, survey 2; no. $00875 / 2015$, survey 3 ).

Intake24 administration. For all three surveys included in the reliability analyses, participants were asked to complete Intake 24 on $4 \mathrm{~d}$ over a $10 \mathrm{~d}$ period, including both week and weekend days. Participants were not aware of their scheduled days in advance but were sent an email on the day of each recall with the URL and log-in details asking them to complete a recall for the previous day's food intake.

Data analysis. For individuals completing four or more recalls using Intake24, we assessed both test-retest reliability of a single recall and reliability of a single-repeat recall; the latter was done by comparing the average of the first two recalls (pair 1) against the average for the following two recalls (pair 2). For both methods, intra-class correlation coefficients (ICC) and their 95\% CI were calculated using a two-way mixed-effects model for absolute agreement; this included evaluation of the influence of age and sex on reliability. Reliability is classified as poor, moderate, good or excellent based on the CI of the ICC as recommended by Koo \& $\mathrm{Li}^{(24)}$. In addition, the method of Bland \& Altman was used to assess agreement (with $95 \%$ limits) between the first and second recall, and between the average of first two recalls and the average of following two recalls. The ratios of reported intakes were calculated for energy and key nutrients. As the data were not normally distributed they 
Table 1. Baseline characteristics of participants completing the doubly labelled water (DLW) study*

(Mean values and standard deviations; minimum and maximum values)

\begin{tabular}{|c|c|c|c|c|}
\hline & Mean & SD & Minimum & Maximum \\
\hline Age (years) & $54 \cdot 3$ & $7 \cdot 30$ & $40 \cdot 0$ & 65.0 \\
\hline Height (cm) & $171 \cdot 2$ & 9.45 & $150 \cdot 6$ & 194.4 \\
\hline Weight (kg) & $78 \cdot 2$ & $13 \cdot 75$ & 48.7 & $110 \cdot 8$ \\
\hline BMI $\left(\mathrm{kg} / \mathrm{m}^{2}\right)$ & $26 \cdot 6$ & 3.47 & $20 \cdot 4$ & 36.6 \\
\hline DLW TEE $(\mathrm{kJ} / \mathrm{d})$ & 12007 & 2374.9 & 6852 & 17043 \\
\hline El 1 d (kJ/d) & 8885 & 4287 & 1622 & 21956 \\
\hline EI 2 d (kJ/d) & 9261 & 4019 & 4175 & 21979 \\
\hline El $3 \mathrm{~d}(\mathrm{~kJ} / \mathrm{d})$ & 9240 & 4008 & 4520 & 16962 \\
\hline $\mathrm{kO}(/ \mathrm{d})$ & 0.119 & 0.030 & 0.066 & 0.257 \\
\hline $\mathrm{kH}(/ \mathrm{d})$ & 0.093 & 0.028 & 0.044 & 0.228 \\
\hline $\mathrm{NO}$ (mol) & 2118 & 435 & 1215 & 3131 \\
\hline $\mathrm{NH}(\mathrm{mol})$ & 2180 & 448 & 1251 & 3224 \\
\hline
\end{tabular}

TEE, total energy expenditure; $\mathrm{El}$, energy intake; $\mathrm{kO}$, decay constant for $\mathrm{O}$; $\mathrm{kH}$, decay constant for $\mathrm{H}$; $\mathrm{NO}$, body oxygen pool; $\mathrm{NH}$, body hydrogen pool.

${ }^{*}$ The mean of the observed space ratio was 1.033 (range 1.016-1.046).

were log-transformed. The values presented are the ratios of the geometric mean. All analyses were conducted with SPSS (SPSS Statistics for Windows, version 22.0; IBM Corp.) or R $(\mathrm{v} 3.4 .3)^{(25)}$ statistical software packages. $P$ values were considered statistically significant at the $\alpha=0.05$ level.

\section{Results}

Validation of Intake24 against doubly labelled water measures of total energy expenditure

A total of ninety-eight participants (fifty women and forty-eight men) completed at least one $24 \mathrm{~h}$ recall (Table 1 ). Demographic data for participants completing two recalls and three recalls varied only slightly. Participants ranged in age from 40 to 65 years, and had a mean BMI of $26 \cdot 6$ (range 20-37) $\mathrm{kg} / \mathrm{m}^{2}$, with no significant change in weight over the recording period. The mean weight change for the participants was +0.09 (SD 0.80) kg, eight participants lost between 1 and $1.5 \mathrm{~kg}$ while twelve participants gained between 1 and $2.2 \mathrm{~kg}$. The remaining seventy-five participants had a weight difference of less than $1 \mathrm{~kg}$ between the beginning and the end of the data collection period.

The mean of EI from three recalls and DLW-based TEE were $9240(\mathrm{sD} \quad 4008 \cdot 5)$ and $11670(\mathrm{sD} \quad 2279 \cdot 8) \mathrm{kJ} / \mathrm{d}$, respectively, indicating under-estimation of self-reported EI by $25 \%$ and almost twofold greater variation of self-reported EI in the population (SD $4008.5 v .2279 .8 \mathrm{~kJ} / \mathrm{d}$ ). Although reporting accuracy of the population averages did not appear to change markedly with increasing number of days recalled, the precision, as evidenced by the width of the limits of agreement (Fig. 1), improved with number of recalls.

The Bland-Altman plots (Fig. 1) show a range of underestimation and over-estimation of EI reported using Intake24 amongst the individuals in this validation study. There is some evidence of systematic bias with an increased tendency to under-report at lower levels of intake and to overreport at higher levels of intake when EI based on a single recall is considered (Fig. 1(a)), but this pattern is no longer apparent when the mean of three recalls is used (Fig. 1(c)).

The mixed-effects model indicated no significant pattern for under- or over-reporting across BMI and sex (Table 2 and Supplementary Table S1). Age was positively associated with EI/TEE, indicating that older participants tended to underreport to a lesser extent. On average people of 40 years of age were found to under-report their EI by $42.6 \%$ whereas in people of 60 years of age EI was under-reported by $18.7 \%$.

Scatterplots showing EI against TEE, on both original and $\log$ scales, are provided in Supplementary Fig. S3. The correlations were $0.31,0.42$ and 0.31 for the first, first two and first three recalls (Table 2), respectively, and generally stronger in normal-weight individuals ( 0.69 for first two recalls) than in over-weight $(0 \cdot 23)$ and obese $(0 \cdot 17)$. The deattenuated correlation coefficients after log-transformation are 0.31 for the first recall, 0.47 for the first two recalls and 0.39 for the first three recalls, showing slight improvement after accounting for intra-individual variation.

\section{Assessment of Intake24 reliability}

As data for the reliability analysis are pooled from several separate studies, the number of participants in each age and sex group were not balanced (Table 3).

For most nutrients, considering the mean of a pair of recalls increased the reliability compared with a single recall administration. Pairs of two recalls produced similar population averages for energy and the macronutrients, as evidenced by mean ratios ranging from 0.99 to $1 \cdot 10$. Slightly poorer (a)

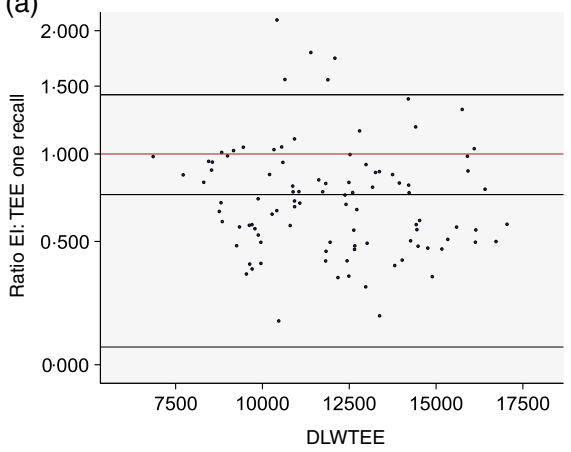

(b)

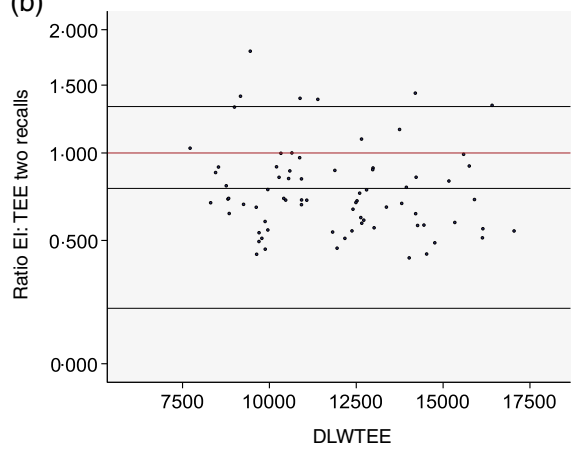

(c)

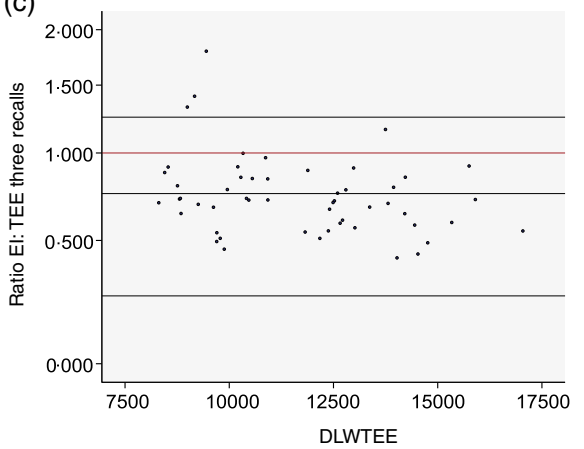

Fig. 1. Bland-Altman plots of ratio of reported energy intake (EI) from first $24 \mathrm{~h}$ recall (a), mean of first two $24 \mathrm{~h}$ recalls (b) and mean of first three $24 \mathrm{~h}$ recalls (c) to total energy expenditure measured by doubly labelled water (DLWTEE). 
Table 2. Accuracy and precision of energy intakes reported using Intake24 - doubly labelled water study*

\begin{tabular}{|c|c|c|c|c|c|c|c|c|c|c|c|c|}
\hline & \multirow{2}{*}{$\begin{array}{l}\text { No. of } \\
\text { recalls }\end{array}$} & \multirow[b]{2}{*}{$n$} & \multicolumn{2}{|c|}{$\begin{array}{l}\text { Energy intake } \\
(\mathrm{kJ} / \mathrm{d})\end{array}$} & \multicolumn{2}{|c|}{ TEE (kJ/d) } & \multirow{2}{*}{$\begin{array}{l}\text { Correlation REI } \\
\text { and TEE }\end{array}$} & \multirow[b]{2}{*}{$\mathrm{P}$} & \multirow{2}{*}{$\begin{array}{l}\text { Mean ratiof } \\
\text { El:TEE }\end{array}$} & \multirow{2}{*}{$\begin{array}{c}\text { Geometric } \\
\text { mean }\end{array}$} & \multicolumn{2}{|c|}{$\begin{array}{c}95 \% \text { Limits of } \\
\text { agreement } \neq\end{array}$} \\
\hline & & & Mean & SD & Mean & SD & & & & & Lower & Upper \\
\hline \multirow[t]{3}{*}{ All } & 1 & 98 & 8885 & 4287 & 12006 & 2374 & 0.31 & 0.002 & 0.75 & 0.68 & 0.27 & 1.68 \\
\hline & 2 & 74 & 9261 & 4019 & 11883 & 2351 & 0.42 & $<0.001$ & 0.78 & 0.74 & 0.39 & 1.41 \\
\hline & 3 & 53 & 9240 & 4008 & 11670 & 2279 & 0.31 & 0.025 & 0.75 & 0.72 & 0.40 & $1 \cdot 28$ \\
\hline \multirow[t]{3}{*}{ Men§ } & 1 & 48 & 9799 & 4772 & 13629 & 1934 & 0.22 & 0.14 & 0.72 & 0.64 & 0.23 & 1.81 \\
\hline & 2 & 34 & 10123 & 4185 & 13586 & 1950 & 0.29 & 0.10 & 0.80 & 0.76 & 0.39 & 1.46 \\
\hline & 3 & 24 & 10190 & 4130 & 13306 & 1877 & 0.19 & 0.37 & 0.73 & 0.70 & 0.41 & $1 \cdot 21$ \\
\hline \multirow[t]{3}{*}{ Women } & 1 & 50 & 8008 & 3598 & 10450 & 1588 & 0.25 & 0.082 & 0.77 & 0.72 & 0.34 & 1.52 \\
\hline & 2 & 40 & 8434 & 3708 & 10435 & 1562 & 0.17 & 0.30 & 0.76 & 0.73 & 0.38 & 1.37 \\
\hline & 3 & 29 & 8329 & 3703 & 10317 & 1601 & 0.07 & 0.71 & 0.77 & 0.73 & 0.39 & $1 \cdot 36$ \\
\hline \multirow[t]{3}{*}{$\mathrm{BMI}<25 \mathrm{~kg} / \mathrm{m}^{2} \S$} & 1 & 35 & 8963 & 4357 & 10818 & 2273 & 0.36 & 0.036 & 0.83 & 0.76 & 0.30 & 1.93 \\
\hline & 2 & 31 & 9250 & 4131 & 10916 & 2171 & 0.69 & $<0.001$ & 0.79 & 0.77 & 0.48 & $1 \cdot 24$ \\
\hline & 3 & 23 & 9267 & 4144 & 10657 & 1956 & 0.75 & $<0.001$ & 0.77 & 0.76 & 0.50 & $1 \cdot 15$ \\
\hline \multirow[t]{3}{*}{ BMI 25-30 kg/m² } & 1 & 47 & 7942 & 3174 & 12332 & 2157 & 0.26 & 0.073 & 0.65 & 0.60 & 0.27 & 1.34 \\
\hline & 2 & 32 & 8697 & 3638 & 12257 & 2236 & 0.23 & 0.21 & 0.80 & 0.74 & 0.35 & 1.57 \\
\hline & 3 & 21 & 8717 & 3631 & 11861 & 2022 & -0.19 & 0.42 & 0.78 & 0.73 & 0.36 & 1.49 \\
\hline \multirow[t]{3}{*}{$\mathrm{BMI}>30 \mathrm{~kg} / \mathrm{m}^{2}$} & 1 & 16 & 11487 & 5911 & 13652 & 1992 & 0.27 & 0.31 & 0.84 & 0.74 & 0.26 & $2 \cdot 12$ \\
\hline & 2 & 11 & 10944 & 4602 & 13520 & 2099 & 0.17 & 0.61 & 0.71 & 0.67 & 0.33 & 1.36 \\
\hline & 3 & 9 & 10721 & 4618 & 13817 & 2192 & 0.56 & 0.12 & 0.62 & 0.60 & 0.36 & 1.00 \\
\hline \multirow[t]{3}{*}{ 40-49 years§ } & 1 & 30 & 8217 & 4043 & 12194 & 2326 & 0.15 & 0.44 & 0.69 & 0.62 & 0.25 & 1.57 \\
\hline & 2 & 23 & 8683 & 4894 & 12143 & 2271 & 0.27 & 0.21 & 0.67 & 0.65 & 0.36 & $1 \cdot 15$ \\
\hline & 3 & 15 & 7499 & 3214 & 11752 & 2247 & 0.54 & 0.04 & 0.65 & 0.63 & 0.36 & 1.08 \\
\hline \multirow[t]{3}{*}{$50-59$ years } & 1 & 34 & 8952 & 4865 & 11977 & 2451 & 0.29 & 0.10 & 0.75 & 0.67 & 0.26 & 1.73 \\
\hline & 2 & 25 & 9021 & 5395 & 11767 & 2616 & 0.43 & 0.03 & 0.74 & 0.70 & 0.38 & $1 \cdot 29$ \\
\hline & 3 & 19 & 7455 & 1637 & 12026 & 2535 & 0.47 & 0.04 & 0.67 & 0.66 & 0.44 & 1.00 \\
\hline \multirow[t]{3}{*}{$60-65$ years } & 1 & 34 & 9410 & 3913 & 11872 & 2402 & 0.50 & 0.002 & 0.79 & 0.74 & 0.32 & 1.71 \\
\hline & 2 & 26 & 11298 & 5346 & 11764 & 2223 & 0.23 & 0.26 & 0.92 & 0.88 & 0.48 & 1.59 \\
\hline & 3 & 19 & 10395 & 4114 & 11251 & 2083 & 0.05 & 0.85 & 0.91 & 0.87 & 0.49 & 1.54 \\
\hline
\end{tabular}

TEE, total energy expenditure; REI, reported energy intake; El, energy intake.

* Data are nested with respect to number of recalls (first recall results for everyone, first two recall results for everyone with at least two recalls, and so on).

$\dagger$ The ratio is the reported mean daily energy intake divided by the total energy expenditure as measured by doubly labelled water. The ratio equal to 1 would indicate exact agreement; $<1$, underestimation; and $>1$, overestimation.

$\ddagger$ Derived from \pm 2 SD of log-transformed ratios.

$\S P=0.11$ for the association of $\mathrm{BMI}$ with the ratio of reported EI to TEE; $P=0.91$, sex difference; and $P=0.003$, age.

reliability was seen for non-milk extrinsic sugars (NMES), alcohol and vitamin C. The limits of agreement were wider for those nutrients for which intakes tend to vary more day to day. The very large limits of agreement for alcohol were due to the fact that most recall days did not include any alcohol and most participants drank on only one of the four recall days, if at all (Table 4).

Summaries of each nutrient for each age group and for all participants are given in Supplementary Table S2. The columns 'lower' and 'upper' refer to the lower and upper quartiles, respectively, of the corresponding nutrient.

Table 3. Demographics of participants included in the reliability study (data from three studies*)

(Numbers of participants)

\begin{tabular}{lrrr}
\hline Age group & Number & Men & Women \\
\hline $11-16$ years & 87 & 34 & 53 \\
17-24 years & 124 & 80 & 44 \\
25-64 years & 68 & 31 & 37 \\
65 years + & 24 & 18 & 6 \\
Total & 303 & 163 & 140 \\
\hline
\end{tabular}

* Test-retest reliability of energy and nutrient intakes was assessed using data from three further UK-based studies where participants aged 11 to 88 years completed Intake24 a minimum of four times, as described in the Methods section.
Supplementary Table S3 shows how agreement varied by age group. The pairs of $24 \mathrm{~h}$ recalls gave values within $10 \%$ of each other for energy and macronutrients, with the exception of alcohol where there was an $8 \%$ difference for the 11 - to 16-year-old group ranging up to a $76 \%$ difference for the 16- to 24-year-old group.

ICC showed poor to moderate agreement in nutrient intakes. ICC for repeatability of a single recall are lower than for two recalls considered together, indicating large intra-individual variation. For example, for reported EI, ICC for a single recall was 0.347 and this increased to 0.516 when the repeatability of $2 \mathrm{~d}$ of recall was considered (Table 4). Alcohol is not included in the ICC analysis due to the large numbers of non-consumers; $86 \%$ of the study population did not consume alcohol during the recording period.

Splitting the data by age group had little influence on the ICC. Supplementary Tables S4 and S5 report the ICC by age group, for a single recall and paired recalls, respectively, according to the model with sex as the only covariate, with $95 \%$ CI, for each nutrient and each age group. ICC show poor to moderate agreement between single recalls for the majority of nutrients, with slight improvement when paired recalls are considered. Repeatability was best in the 65 years and over age group where ICC for intakes of NMES and Fe were moderate to excellent $(0.863$ and 0.857 , respectively) 
and good to excellent ( 0.526 and 0.530 , respectively), for paired recalls and single recalls, respectively.

\section{Discussion}

In comparison with TEE measured in a cohort of UK adults aged 40-65 years, over 10-14 d using DLW, self-reported EI by Intake 24 was underestimated by $25 \%$ on average. The level of under-reporting was similar for men and women but was found to vary significantly with age, with older people tending to under-report to a lesser extent. Comparing the reported EI from a single recall with that from 2 or $3 \mathrm{~d}$ of recall, accuracy did not improve markedly with an increased number of days; however, the precision of estimates did improve, particularly with the second recall. Although accuracy of reporting improved with age, the intra-individual variation in underestimation was constant across age groups. There was some evidence of systematic bias, with an increased tendency to under-report at lower levels of intake and to over-report at higher levels of intake, when reported EI from a single recall is considered, but this pattern disappeared when the mean of three recalls was used. This may be indicative of the day-to-day variation in EI and the need to collect data on multiple days.

Under-reporting of habitual EI may be due to underreporting of food intakes during the recording period, undereating during the recording period or a combination of the two. This has been examined using covert observation of individuals recording their food intake where a reduction in EI (under-eating) of $8 \%$ in men and $3 \%$ in women and underreporting of $9 \%$ by men and $12 \%$ by women combined to give an under-estimate of around $15 \%$ of $\mathrm{EI}^{(26)}$.

Average levels of under-reporting of total EI using Intake24 were similar to traditional dietary assessment methods implemented in surveys in the UK, the USA and elsewhere. The UK National Diet and Nutrition Survey Rolling Programme collects dietary data using a 4-d estimated weight food diary with interview. EI reported using this method was validated in a sub-sample of the population aged 4 years and over (n 371) against TEE assessed using DLW. EI was underestimated on average across all age groups. The lowest levels of under-reporting were seen in the 4- to 10-year-old group where EI was under-estimated by $22 \%$ on average. For participants aged 16 years and over mean under-estimates of EI ranged from 25 to $36 \%{ }^{(27)}$. Lopes et al. ${ }^{(28)}$ compared EI estimated by three interviewer-administered 24-h dietary recalls with TEE measured by DLW in eighty-three adults aged 20-60 years in Brazil. They found EI to be under-estimated by $23 \%$ in men and by $40 \%$ in women. A pooled analysis of five validation studies comparing 24-h dietary recalls with TEE measured by DLW found EI to be under-reported by $15 \%$ on average, ranging from an under-estimate of 28 to $6 \%$ for individual studies ${ }^{(29)}$. Few studies have reported the validity of EI assessed using online dietary systems against DLW-measured TEE. Reported EI based on six 24-h recalls completed using the web-based system DietDay was validated against DLW in 233 adults aged 21-69 years and EI was found to be under-reported by $10 \%$ on average ${ }^{(30)}$. Comparison of a 
4-d web-based food record with DLW-measured TEE in forty middle-aged adults in Sweden found that men under-reported EI by $24 \%$ on average whereas women under-reported by $16 \%{ }^{(31)}$. EI reported using the online dietary recall system ASA $24^{\circledR(5)}$ was compared against DLW-measured TEE in older adults (mean age 62 years for women and 64 years for men) ${ }^{(32)}$. The average under-estimation of EI was $17 \%$ in men $(n$ 485) and $15 \%$ in women $(n 472)$, comparable with the $18.7 \%$ under-estimation in our sample of 60 -year-olds. Validation of EI against TEE using DLW assumes that participants are in energy balance. TEE is measured over a relatively short period, often 10-14 d and the weight change from a 500 $\mathrm{kcal} / \mathrm{d}(2092 \mathrm{~kJ} / \mathrm{d})$ deficit over this period would only be around $500 \mathrm{~g}$. Given the proportion of the population who are over-weight or obese, many participants may be making efforts to reduce their EI. Therefore, an EI:TEE ratio lower than 1.0 could represent accurate EI estimates to some extent.

In a study of 627 adults aged $50-70$ years the reproducibility of a single dietary recall reported using ASA $24^{\circledR}$ was low, with ICC for energy and protein of $0 \cdot 28$ and $0 \cdot 25$, respectively ${ }^{(33)}$, slightly lower than the repeatability of a single recall using Intake24 (0.347 and 0.344$)$. This difference may be due to the longer time between recalls in the ASA $24^{\circledR}$ study where recalls were repeated at 3-month intervals.

Assessing the reliability of measures of EI and nutrients via repeated 24-h recalls is complicated by the genuine day-to-day variation in individual food intakes. A way to address this is to collapse results of multiple recalls into pairs and test their reliability. Results of the Bland-Altman analysis showed good agreement between recalls 1 and 2 and recalls 3 and 4 for energy and macronutrients, but greater variability for alcohol and NMES. This may reflect greater day-to-day variability in intake of these nutrients, indicating that more than two recalls are required for accurate estimation of usual intakes for some nutrients $^{(34)}$. Better reliability of intakes reported using Intake24 was observed in men and those aged 64 years and over, possibly suggesting less day-to-day variability in these individuals' diets.

\section{Study limitations and strengths}

We have conducted a detailed analysis of misreporting of EI including how this varies by age, sex and BMI; however, the findings are not generalisable to people of ethnic minority groups, or from different socio-economic backgrounds, for whom the extent of misreporting may differ. Although the sample size for the DLW validation of Intake24 is relatively large for such studies, there was no consistency in the range of week and weekend days for which participants completed their recalls. The small number of days recalled is also a limitation but may reflect common choices in study designs. Energy expenditure was assessed over a 10-14 d period; for logistic reasons participants were free to choose the days to complete Intake24 and so may have avoided completing recalls on days they considered their intake to be unhealthy or too complicated to report, which would be likely to be days that EI was high.

TEE estimated using DLW requires a number of assumptions and inferences. These include that the individual is weight stable and that the levels of background isotope intake remain constant ${ }^{(35,36)}$. In this study, two pre-dose samples were taken which will reduce the associated error assigned to the variation in natural abundance ${ }^{(36)}$. Furthermore, as DLW only measures $\mathrm{CO}_{2}$ production and not directly $\mathrm{O}_{2}$ consumption, some knowledge of the energy equivalent of $\mathrm{CO}_{2}$ is needed for TEE estimation. This can be highly variable and macronutrient dependent. In the absence of a measurement of the respiratory quotient (RQ) which allows determining macronutrient oxidation, RQ was assumed to be 0.85 , it being the average RQ of a standard Western diet. The RQ based on the dietary intake reported by our participants was 0.849 on average (SD 0.021) and given the known issues with under-reporting of food intakes rather than make any assumptions around the nutritional composition of 'missing foods' the fixed RQ was used. In total, the error associated with our calculations $(2.04 \pm 0.76 \%)$ is well within the $2-8 \%$ error deemed acceptable using the DLW method ${ }^{(37)}$.

Assessment of the repeatability of any short-term measure of dietary intake is complicated by the true day-to-day variation in individual intake. In our study we did not directly determine how much of the variation would be due to measurement error or true variability of food intakes. What the reliability results do indicate is the degree to which $2 \mathrm{~d}$ of recall is sufficient to obtain an estimate of habitual intake for a particular nutrient. At the population level, reported intakes of energy and macronutrients from one pair of non-consecutive $24 \mathrm{~h}$ recalls were within $10 \%$ of those reported in a further pair of recalls completed by the same individual. At the individual level, however, there is much greater variation as evidenced by the wide limits of agreement and low ICC. However, week and weekend days were not balanced across the recall pairs, and therefore reliability estimates may be slightly attenuated for this reason. The repeatability analysis is conducted on pooled data from three studies that covered different age groups; while this is a strength in terms of generalisability of results, this also increases between-individual variation by which pooled ICC may be overestimated.

\section{Conclusions}

Under-reporting of EI is a consistent finding when using dietary assessment methods which rely on self-reports of food and drink intake. We report that EI reported using Intake24 were under-estimated by around $25 \%$ compared with TEE and were only weakly correlated.

From the reliability study, our findings indicate that $2 \mathrm{~d}$ of recalls using Intake24 are sufficient for the assessment of habitual intake of energy and macronutrients at the group level. More days are likely to be required for food components where day-to-day variation is greater, especially alcohol. The underestimation of EI using Intake24 is comparable with more intensive methods of data collection such as interviewer-led $24 \mathrm{~h}$ recalls or estimated food diaries. As data are collected remotely, without the need for trained interviewers, and participants can complete recalls at a time convenient for them, the system offers a reduced cost and burden alternative for collecting dietary intake information. Future work should focus on whether the 
validity of self-reported methods such as Intake24 can be improved by combining these with image capture-based methods ${ }^{(38-40)}$ and/or mathematical modelling ${ }^{(41,42)}$.

\section{Supplementary material}

The supplementary material for this article can be found at https://doi.org/10.1017/jns.2019.20.

\section{Acknowledgements}

We would like to thank Lewis Griffiths, Nicola Kimber, Eoin McNamara, Katie Palmer and Richard Salisbury (MRC Epidemiology Unit, Cambridge) for their contributions to the recruitment and data collection for the DLW validation study. We also thank the co-ordinators, principal investigators and operational teams working on the Fenland Study. From the MRC Elsie Widdowson Laboratory, Cambridge, Polly Page and Toni Steer are acknowledged for their input and advice regarding the National Diet and Nutrition Survey, and Priya Singh, Elise Orford and Kevin Donkers (MRC Elsie Widdowson Laboratory, Cambridge) are acknowledged for their help with isotope dose preparation and urine sample enrichment analysis for the DLW validation study. Eimear Duffy conducted all aspects of survey 2 included in the reliability analysis. Shanna Christie and ScotCen conducted the recruitment for survey 3 and Lorraine Murray and IPSOSMORI conducted the recruitment for survey 1 included in the reliability analysis. Our sincere thanks also go to the volunteers who participated in each study.

UK Medical Research Council support is acknowledged by S. B., S. E. H. and K. L. W. (MC UU 12015/3), by F. I. and N. G. F. (MC UU 12015/5), N. W. (MC UU 12015/1) and M. C. V. (MC U105960384). S. B., K. L. W., N. G. F. and N. W. also acknowledge National Institute for Health Research (NIHR) Biomedical Research Centre Cambridge: Nutrition, Diet, and Lifestyle Research Theme (IS-BRC-1215-20014). A. J. A. is funded by NIHR as an NIHR Research Professor and is a member of FUSE. Cost of isotope work was part funded by a grant from MedImmune Ltd to S. B., part funded by Newcastle University. Food Standards Scotland (previously Food Standards Agency Scotland) funded study 1 and study 3 which are included in the reliability analysis.

E. F., F. I., S. E. H., K. L. W., P. O., N. W., N. G. F. and S. B. designed the research; E. F., S. E. H., K. L. W., M. C. V., M. K. R., J. C. B., E. L. S. and N. G. F. conducted the research; I. P. and T. O. developed the Intake24 system; S. E. H. provided essential reagents and materials; E. F., M. C. V., M. K. R., J. C. B. and E. L. S. analysed data; E. F., C. L., F. I. and S. B. designed the statistical analysis; E. F. and C. L. performed statistical. analysis; E. F., C. L., F. I., S. E. H., K. L. W., M. C. V., M. K. R., J. C. B., E. L. S., A. J. A., P. O., N. W., N. G. F. and S. B. wrote the paper; E. F. and S. B. had primary responsibility for final content; all authors read and approved the final manuscript.
There are no conflicts of interest.

\section{References}

1. Black AE (1996) Under-reporting of energy intake at all levels of energy expenditure: evidence from doubly labelled water studies. Proc Nutr Soc 56, $121 \mathrm{~A}$.

2. Goris AHC, Westerterp-Plantenga MS \& Westerterp KR (2000) Undereating and underrecording of habitual food intake in obese men: selective underreporting of fat intake. Am J Clin Nutr 71, 130-134.

3. Livingstone MBE, Prentice AM, Strain JJ, et al. (1990) Accuracy of weighed dietary records in studies of diet and health. Br Med J 300, 708-712.

4. Albar SA, Alwan NA, Evans CE, et al. (2016) Agreement between an online dietary assessment tool (myfood24) and an intervieweradministered 24-h dietary recall in British adolescents aged 11-18 years. BrJ Nutr 115, 1678-1686.

5. Subar AF, Kirkpatrick SI, Mittl B, et al. (2012) The Automated Self-Administered 24-hour dietary recall (ASA24): a resource for researchers, clinicians, and educators from the National Cancer Institute. J Acad Nutr Diet 112, 1134-1137.

6. Touvier M, Kesse-Guyot E, Mejean C, et al. (2011) Comparison between an interactive web-based self-administered $24 \mathrm{~h}$ dietary record and an interview by a dietitian for large-scale epidemiological studies. Br J Nutr 105, 1055-1064.

7. Liu B, Young H, Crowe FL, et al. (2011) Development and evaluation of the Oxford WebQ, a low-cost, web-based method for assessment of previous $24 \mathrm{~h}$ dietary intakes in large-scale prospective studies. Public Health Nutr 14, 1998-2005.

8. Rowland MK, Adamson AJ, Poliakov I, et al. (2018) Field testing of the use of Intake24 - an online 24-hour dietary recall system. Nutrients 10, E1690.

9. Raper N, Perloff B, Ingwersen L, et al. (2004) An overview of USDA's dietary intake data system. J Food Compos Anal 17, 545-555.

10. Roe M, Pinchen H, Church S, et al. (2015) McCance and Widdowson's The Composition of Foods seventh summary edition and updated composition of foods integrated dataset. Nutr Bull 40, 36-39.

11. Foster E, Hawkins A, Barton KL, et al. (2017) Development of food photographs for use with children aged 18 months to 16 years: comparison against weighed food diaries - The Young Person's Food Atlas (UK). PLOS ONE 12, e0169084.

12. Foster E, Matthews JN, Lloyd J, et al. (2008) Children's estimates of food portion size: the development and evaluation of three portion size assessment tools for use with children. Br J Nutr 99, 175-184.

13. Simpson E, Bradley J, Poliakov I, et al. (2017) Iterative development of an online dietary recall tool: INTAKE24. Nutrients 9, E118.

14. Bradley J, Simpson E, Poliakov I, et al. (2016) Comparison of INTAKE24 (an online 24-h dietary recall tool) with interviewer-led 24-h recall in 11-24 year-old. Nutrients 8, E358.

15. Lifson N, Gordon GB \& McClintock R (1955) Measurement of total carbon dioxide production by means of $\mathrm{D}_{2} \mathrm{O}^{18}$. J Appl Physiol 7, 704-710.

16. Schoeller DA \& Van Santen E (1982) Measurement of energy expenditure in humans by doubly labeled water method. $J$ Appl Physiol 53, 955-959.

17. Livingstone MB, Prentice AM, Coward WA, et al. (1992) Validation of estimates of energy intake by weighed dietary record and diet history in children and adolescents. Am J Clin Nutr 56, 29-35.

18. Black AE (2000) Critical evaluation of energy intake using the Goldberg cut-off for energy intake: basal metabolic rate. A practical guide to its calculation, use and limitations. Int J Obes 24, 1119-1130.

19. O'Connor L, Brage S, Griffin SJ, et al. (2015) The cross-sectional association between snacking behaviour and measures of adiposity: The Fenland Study, UK. Br J Nutr 114, 1286-1293.

20. Bland JM \& Altman DG (1986) Statistical methods for assessing agreement between two methods of clinical measurement. Lancet i, 307-310. 
21. Roether W (1970) Water- $\mathrm{CO}_{2}$ exchange set-up for the routine 18oxygen assay of natural waters. Int J Appl Radiat Isot 21, 379-387.

22. Craig H (1957) Isotopic standards for carbon and oxygen and correction factors for mass-spectrometric analysis of carbon dioxide. Geochim Cosmochim Acta 12, 133-149.

23. Rimm EB, Giovannucci EL, Stampfer MJ, et al. (1992) Reproducibility and validity of an expanded self-administered semiquantitative food frequency questionnaire among male health professionals. Am J Epidemiol 135, 1114-1126.

24. Koo TK \& Li MY (2016) A guideline of selecting and reporting intraclass correlation coefficients for reliability research. J Chiropr Med 15, 155-163.

25. R Core Team (2018) R: A Language and Environment for Statistical Computing. Vienna, Austria: R Foundation for Statistical Computing.

26. Stubbs RJ, O'Reilly LM, Whybrow S, et al. (2014) Measuring the difference between actual and reported food intakes in the context of energy balance under laboratory conditions. BrJ Nutr111, 2032-2043.

27. Bates B, Lennox A, Prentice A, et al. (2014) National Diet and Nutrition Survey Results from Years 1, 2, 3 and 4 (Combined) of the Rolling Programme (2008/2009-2011/2012). A survey carried out on behalf of Public Health England and the Food Standards Agency. https://www.food.gov.uk/sites/default/files/media/ document/ndns-appendix-x.pdf (accessed July 2019).

28. Lopes TS, Luiz RR, Hoffman DJ, et al. (2016) Misreport of energy intake assessed with food records and 24-h recalls compared with total energy expenditure estimated with DLW. Eur J Clin Nutr 70, 1259-1264.

29. Freedman LS, Commins JM, Moler JE, et al. (2014) Pooled results from 5 validation studies of dietary self-report instruments using recovery biomarkers for energy and protein intake. $A m J$ Epidemiol 180, 172-188.

30. Arab L, Tseng C-H, Ang A, et al. (2011) Validity of a multipass, web-based, 24-hour self-administered recall for assessment of total energy intake in blacks and whites. Am J Epidemiol 174, 1256-1265.

31. Nybacka S, Forslund HB, Wirfält E, et al. (2016) Comparison of a web-based food record tool and a food-frequency questionnaire and objective validation using the doubly labelled water technique in a Swedish middle-aged population. J Nutr Sci 5, e39.
32. Park Y, Dodd KW, Kipnis V, et al. (2018) Comparison of selfreported dietary intakes from the Automated Self-Administered 24-h recall, 4-d food records, and food-frequency questionnaires against recovery biomarkers. Am J Clin Nutr 107, 80-93.

33. Yuan C, Spiegelman D, Rimm EB, et al. (2017) Relative validity of nutrient intakes assessed by questionnaire, 24-hour recalls, and diet records as compared with urinary recovery and plasma concentration biomarkers: findings for women. Am J Epidemiol 187, 1051-1063.

34. Basiotis PP, Welsh SO, Cronin FJ, et al. (1987) Number of days of food intake records required to estimate individual and group nutrient intakes with defined confidence. I Nutr 117, 1638-1641.

35. Coward WA \& Cole TJ (1990) The doubly labeled water method for the measurement of energy expenditure in humans: risks and benefits. In Bristol-Myers Nutrition Symposia 1990, vol. 9, pp. 139176. New York: Academic Press, Inc.

36. Ritz P, Cole TJ, Couet C, et al. (1996) Precision of DLW energy expenditure measurements: contribution of natural abundance variations. Am J Physiol Endocrinol Metab 270, E164-E1E9.

37. Schoeller DA (1988) Measurement of energy expenditure in free-living humans by using doubly labeled water. J Nutr 118, 1278-1289.

38. Martin CK, Han H, Coulon SM, et al. (2009) A novel method to remotely measure food intake of free-living individuals in real time: the remote food photography method. Br J Nutr 101, 446-456.

39. Schap TE, Zhu F, Delp EJ, et al. (2014) Merging dietary assessment with the adolescent lifestyle. J Hum Nutr Diet 27, 82-88.

40. Sun M, Burke LE, Baranowski T, et al. (2015) An exploratory study on a chest-worn computer for evaluation of diet, physical activity and lifestyle. J Healthc Eng 6, 1-22.

41. Sanghvi A, Redman LM, Martin CK, et al. (2015) Validation of an inexpensive and accurate mathematical method to measure longterm changes in free-living energy intake, 2. Am J Clin Nutr 102, 353-358.

42. Tooze JA, Midthune D, Dodd KW, et al. (2006) A new statistical method for estimating the usual intake of episodically consumed foods with application to their distribution. J Am Diet Assoc 106, 1575-1587. 